Mappemonde

Revue trimestrielle sur l'image géographique et les

formes du territoire

$124 \mid 2018$

Varia

\title{
Des marmottes et des hommes
}

\section{Émilie Baudat et Laine Chanteloup}

URL : http://journals.openedition.org/mappemonde/377

DOI : $10.4000 /$ mappemonde.377

ISSN : 1769-7298

Éditeur

UMR ESPACE

\section{Référence électronique}

Émilie Baudat et Laine Chanteloup, «Des marmottes et des hommes », Mappemonde [En ligne], 124 |

2018, mis en ligne le 10 juillet 2018, consulté le 15 septembre 2020. URL : http://

journals.openedition.org/mappemonde/377

Ce document a été généré automatiquement le 15 septembre 2020.

\section{c) (i) (2)}

La revue Mappemonde est mise à disposition selon les termes de la Licence Creative Commons Attribution - Pas d'Utilisation Commerciale - Partage dans les Mêmes Conditions 4.0 International. 


\title{
Des marmottes et des hommes
}

\author{
Émilie Baudat et Laine Chanteloup
}

Des marmottes et des hommes

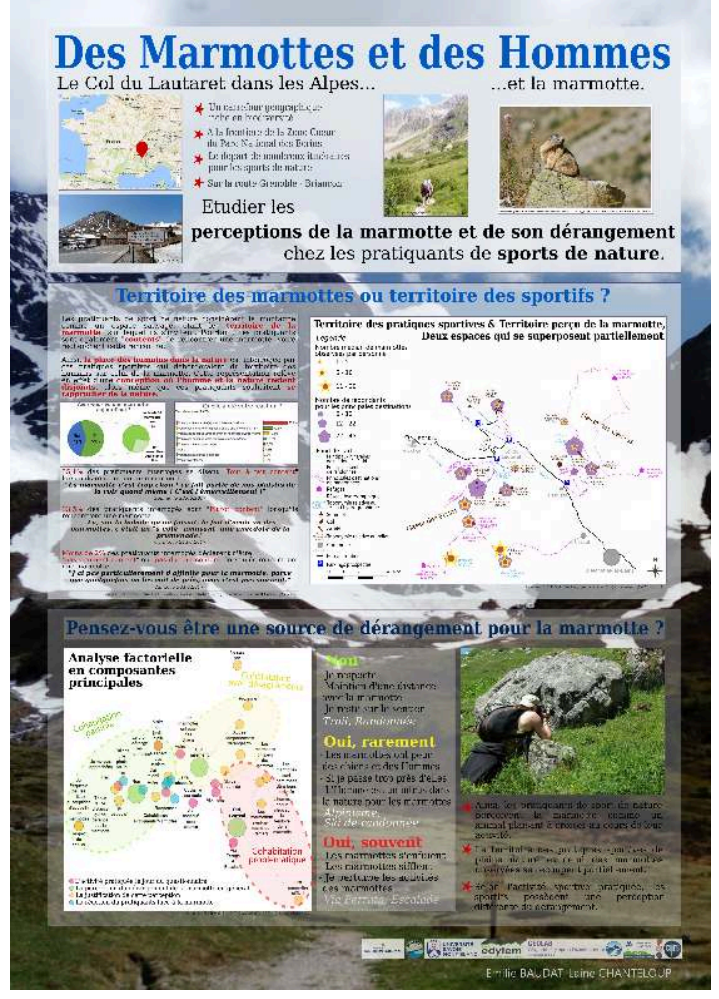

1 Dérangées par les touristes, voire nourries, les marmottes montrent des modifications de leur comportement face au développement des activités récréatives et touristiques en montagne: elles passent par exemple plus de temps à la surveillance et moins de temps à fourrager, et semblent également diminuer leur vigilance à l'égard de l'humain (Louis et Le Berre, 1995 ; Griffin et al. 2007). Or, la marmotte est le symbole de la faune sauvage alpine, facile à observer et attachante car propice à l'anthropomorphisme (Fourcade, 1995). L'augmentation continue du nombre de pratiquants en montagne 
venus assouvir un désir de nature pose donc la question de leur impact sur cette faune sauvage emblématique.

2 Le poster présente les résultats de l'enquête réalisée auprès des pratiquants de sports de nature et des professionnels de la montagne au col du Lautaret de mars à juillet 2017. Elle est composée d'une étude quantitative réalisée à partir de 500 questionnaires passés auprès des pratiquants de sports et d'une partie qualitative fondée sur 12 entretiens de professionnels et de sportifs.

Plus de la moitié des répondants au questionnaire ont vu des marmottes et la majorité se déclarent « tout à fait content » d'en apercevoir. Cette rencontre constitue donc « un plus » à la sortie sportive, voire un but pour certains randonneurs. La superposition des observations de marmottes par les répondants et du nombre de répondants par parcours permet de voir que les territoires de pratiques sportives et de vie des marmottes se recoupent en partie. Ainsi, même si certains itinéraires sont sélectionnés pour «aller voir les marmottes », les espaces les plus fréquentés par les pratiquants ne sont pas toujours ceux où le maximum de marmottes est observé. Dès lors, comment les pratiquants perçoivent-ils cette proximité, quelles sont leurs perceptions et la représentation de leur impact pour les populations de marmottes?

4 Une typologie de ces perceptions peut être dressée à partir de l'ACP (Analyse en Composantes Principales) croisant quatre variables issues du questionnaire: la perception du dérangement de la marmotte en général, la justification de cette perception, la réaction du pratiquant face à la marmotte et l'activité pratiquée dans la journée. Trois profils émergent alors :

- Des pratiquants estimant que la cohabitation avec la marmotte est paisible (en vert sur le graphique) avec principalement des pratiquants de randonnée, les pratiquants de trail et les visiteurs du jardin alpin. Ils déclarent respecter les sentiers et les terriers et maintenir une certaine distance avec la marmotte. Cependant, la confiance en cette cohabitation permet même à certains répondants de s'approcher de la marmotte. En présence d'une marmotte, ils l'observent, voire la prennent en photo ou en film.

- Des pratiquants qui se représentent une cohabitation avec désagréments pour la marmotte (en jaune). Ce sont surtout les pratiquants de ski de randonnée, d'alpinisme et de cyclisme sur route. Pour eux, c'est avant tout la proximité avec l'animal sauvage qui peut occasionner son dérangement, ils estiment en particulier que les marmottes ont peur des humains et de leurs chiens. Ils se considèrent comme dérangeants pour la marmotte car l'humain serait un intrus dans son environnement naturel et la perturberait dans ses activités.

- Des pratiquants pour lesquels la cohabitation Homme/marmotte semble problématique (en rouge). Ce sont surtout des pratiquants de via ferrata et d'escalade. Ils se perçoivent comme dérangeants pour les marmottes car ils les entendent siffler et les voient s'enfuir. Ils considèrent aussi qu'ils perturbent leurs activités et que les marmottes ont peur des humains.

5 Cette étude permet donc d'envisager certains rapports humains-animaux dans l'espace montagnard. Les territoires de pratique sportive et celui des marmottes ne superposent pas totalement au col du Lautaret, cependant, les pratiquants interrogés considèrent la montagne comme étant davantage le territoire de la marmotte que celui de la pratique sportive. S'ils se considèrent globalement comme de potentiels intrus dans l'espace montagnard, les perceptions varient en fonction des sports pratiqués et de la réaction de la marmotte observée lors de la rencontre. 


\section{BIBLIOGRAPHIE}

FOURCADE P. (1995). « Figuration et statut de la marmotte dans les usages sociaux du milieu alpin ». Journal d'agriculture traditionnelle et de botanique appliquée, vol. 37, n 1, p. 75-88.

GRIFFIN S. C., VALOIS T., TAPER M. L., SCOTT MILLS L. (2007). « Effects of Tourists on Behavior and Demography of Olympic Marmots ». Conservation Biology, vol. 21, n 4, p. 1070-1081.

LOUIS S., LE BERRE M. (1996). « Impact de la pression anthropique sur la marmotte alpine ». In M. LE BERRE et R. RAMOUSSE (éd.), $3^{e}$ Journée d'étude sur la marmotte alpine. Lyon (Villeurbanne), 20 décembre 1995. Actes du séminaire national. Villeurbanne (Laboratoire de socioécologie et conservation), p. 59-66.

\section{AUTEURS}

\section{ÉMILIE BAUDAT}

BIOTERRE, Université Paris 1-Panthéon Sorbonne

\section{LAINE CHANTELOUP}

EDYTEM, Université Savoie Mont Blanc, GEOLAB, Université de Grenoble Joseph Fourier 